\title{
La métaphysique du bien selon I' acception de St. Augustin
}

L'antique penseur chrétien de l'Occident, dit doctor gratiae, ne niait, pas -contrairement à certaines opinions- la valeur de la nature. On en trouve la preuve dans sa théorie du bien à multiples aspects: théologique, éthique, épistemologique et ontique. Dans nos considérations présentes, nous nous penchons sur la métaphysique du bien, chez St. Augustin, et en particulier, sur la conception ontologique du bien ontique. Evidemment, St. Augustin n'a pas formulé explicitement la métaphysique du bien, mais il est possible de la reconstruire à la lumière de l'analyse de ses nombreuses déclarations détaillées ${ }^{1}$. Il y a una abondante littérature analysant les élements philosophiques de l'augustinisme ${ }^{2}$. Certaines conceptions (entre autres, celles de la vérité, du temps, de la substance) font l'objet d'amples études monographiques, mais nous manquons toujours de travaux qui discuteraient ex professo la théorie du bien ontique ${ }^{3}$. Quoiqu'il existe des oeuvres vastes et précieuses démontrant l'axiologie de St. Au-

1. En ce qui concerne le caractère de la philosophie augustínienne, cf. F. CAYRE, Initiation à la philosophie de saint Augustin (Paris 1947) 8$13,39-43,245-249$.

2. Une bibliographie se trouve chez F. KöRNER, Das Sein und der Mensch. Die existenzielle Seinsentdeckung des jungen. Augustin (München 1959) 1-26. Cf. M. F. SCIACCA, L'intériorité objective, (Milano 1953). J. F. ANDERSon, St. Augustine and Being. A metaphysical Essay, (The Hague 1965).

3. Les auteurs suivants parlent de ce problème plutôt marginalement: Fr. J. von RINTELEN, Deus bonum omnis boni, dans: Aurelius Augustinus (Köln 1930) 206-216; FR. J. voN RINTELEN, Augustinus-Enalichkeit und Unendlichkeit des Guten / bonum /, "Horizont" 10 - 1967-, 70-80; R. Jolivet, Le problème du mal d'après Saint Augustin (Paris 1936); A. A. Moon, The De natura boni of Saint Augustine. A Translation with an Introduction and Commentary (Washington 1955) 31-40. D'autres ouvrages traitant de l'axiologie de St. Augustin donnent des opinions encore plus succinctes sur la nature du bien ontique. 
gustin, son message philosophique n'y joue, en de nombreux cas, qu' un rôle d'inspiration ".

Il est aisé de comprendre la métaphysique du bien chez St. Augustin à la lumière de ses expériences personnelles. Au cours de a jeunesse, et notamment des années $372-383$, il avait été sympathisant du manichéisme, ${ }^{5}$, doctrine fondée au début du IIle siècle apr. J.- Chr. par Manès. Le manichéisme présentait un assemblage d'éléments pris au christianisme, au judaïsme, au mithraïsme et au chaldéisme ${ }^{\circ}$. C'était une conception dualiste du monde et de l'homme, admettant l'existence de deux principes éternels et également puissants en tant que pré-éléments du réel: le bien et le mal. Le bien est Dieu, tout être spirituel et la lumière; le mal est incorporé dans satan, la matière et les ténèbres. Une telle vision du monde comprenait des aspects théologiques ainsi que philosophiques, et dans l'aspect philosophique, elle présentait une acceptation de l'axiologie du dualisme. Après avoir abandonné le manichéisme, Augustin subit l'influence de la philosophie platonicienne et néo-platonicienne ${ }^{7}$. Il lit alors les Ennéades où l'attribut du bien dans la nature divine est fortement accentué. L'influence du néo-platonisme et de la Révélation chrétienne (de la Bible) ont amené Augustin à une métamorphose radicale d'opinions, ce qui trouva son expression également dans sa nouvelle conception du bien ontique. Cette conception est incluse dans les traités anti-manichéens, dont les plus importants sont: Contra Faustum Manichaeum, De moribus Manichaeorum, De natura

4. Les oeuvres de l'augustinien allemand $J$. Hessen en sont un exemple classique. Cf. ses ouvrages: Wertphilosophie (Paderborn 1937); Die Werte des Heiligen. Eine neue Religionsphilosophie, (Regensburg 1938).

5. Cf. P. AlFARIC, L'évolution intellectuelle de Saint Augustin. Du Manichéisme au Néoplatonisme (Paris 1918), p. $394 \mathrm{ss}$; F. Körner, Das Sein und der Mensch, éd. cit., p. 76-88. H. I. MARRou accentue la nécessité d'expliquer les oeuvres d'Augustin dans le contexte de sa biographie et dēs conditions historiques générales; cf. Saint Augustin et la fin de la culture antique (Paris 1958).

6. H. C. PuEch, Le manichéisme, son fondateur, sa doctrine (Paris 1949); S. Petrement, Le dualisme chez Platon, les gnostiques et les manichéens (Paris 1947); G. QuISPEL, Gnosis als Weltreligion (Zürich 1951); A. A. Moon, The De natura boni of Saint Augustine, éd. cit., 14-30.

7. L'influence de Plotin avait été la plus essentielle, Augustin avait lu d'importants fragments des Ennéades de Plotin. Cf. L. Grandgeorge, Saint Augustin et le Néoplatonisme (Paris 1896) p. 36-40; F. CAYre, Initiation à la philosophie de Saint Augustin, éd. cit., p. $80 \mathrm{ss}$ Actuellement, on désigne souvent Porphyre comme intermédiaire entre Plotin et Augustin. Cf. J. O'Mrara, Porphyry's Philosophy from Oracles in Augustine (Paris 1959). 
boni ${ }^{8}$. Les oeuvres ultérieures ne contienent pas de modifications essentielles de l'axiologie augustienne.

\section{La nature du bien ontique}

En rompant avec le dualisme manichéen, Augustin admit le bien en tant qu'élément prédominant et matière du réel. C'etait là une attitude d'optimisme axiologique se ramenant à deux thèses, et notamment: I) le bien est plus universel que le mal, 2) le bien est un phénomène positif et le mal n'est que défaut.

En affirmant l'universalité du bien, le penseur chrétien antique se trouvait dans la zone d'influence du pythagoréisme, du néo-platonisme et de la Bible. De Pythagore, il avait emprunté la conviction sur la finalité de la structure du cosmos, ce qui amenait à la thèse que tout ce qui existe est bon de par sa nature?. Plotin, en acceptant la théorie de l'émanationnisme, admit en tant qu'origine des transformations évolutives du monde, la triade: Absolu, esprit et âme. Parmi les attributs essentiels de l'Absolu, il classa l'unicité et le bien. La divinité, l'hyperagathon, est le (commencement) de tout ce qui existe ${ }^{10}$. La dépendance ontique de l'univers du Pré-bien absolu, a amené Plotin à la conclusion que l'être est identique au bien. Cette équivalence de l'être et du bien, et proclamée par la philosophie néo-platonicienne, a été acceptée par St. Augustin "II. Il a trouvé également un fondement pour son optimisme axiologique dans les paroles de la Bible: "Et Dieu vit toutes les choses qu'il avait faites : et elles étaient très bonnes) (Gén I, 3I ${ }^{12}$.

Les influences mentionnées ci-dessus, autant philosophiques que théologiques, ont décidé St. Augustin à admettre la théorie que le bien est aussi universel que l'être. L'affirmation de l'universalité du bien était liée à la délimitation du bien ontique et du bien éthique. Tels auteurs sont d'avis que le penseur ne discernait pas le bien on-

8. Contra Faustum Man, fut écrit en 397-398, De moribus Manichaeorum en 389-390,, De natura boni en 399 (selon l'opinion de M. F. Sciacca) ou en 405 (selon l'opinion de P. Alfaric et de B. Roland-Gosselin).

9. L'influence du pythagoréisme apparaît le plus visiblement dans le traité De ordine. Cf. De cons. Evang. 1,7 PL 34, 1048.

10. Plotin, Ennéades, VI,7,42; VI, 7,23; V, 5,13.

11. On en trouve la confirmation chez A. A. Moon, op. cit., 30.

12. Confes., $13,2,2$ PL 32,845 . 
tique et le bien moral ${ }^{13}$. Il est vrai que certaines parmi ses déclarations ne sont pas précises, mais en principe, il était conscient du caractère distinctif des deux types du bien. D'ailleurs, déjà dans les Ennéades, l'on trouve une distinction -indirecte au moins - du bien ontique et du bien moral. L'Absolu est le bien ontique primordial, l'arete est le bien éthique lié à l'activité humaine dirigée vers la purification intérieure spirituelle ${ }^{\text {tat }}$. La vertu -l'efficience morale de l'homme- est incontestablement un bien, mais ce n'est pas là le bien suprême.

St. Augustin reportait le discernement du bien ontique et du bien éthique autant à Dieu qu'aux créatures. Dieu est le summum borum, soit le bien pur, absolu et simple; dans sa nature, il y a une parfaite identité entre le bien moral et le bien de la nature ${ }^{15}$. Les créatures n'ont plus cette conformité des deux types du bien; il en résulte souvent un désaccord entre le bien ontique et le bien éthique. Ainsi, le corps humain (les éléments dont il se compose, la santé) et les attributs psychiques de l'homme présentent indubitablement un bien ontique. Le bien éthique apparaît lorsque l'homme emploie sa nature pour des objectifs propres; au cas opposé, il commet le mal ${ }^{16}$. L'évêque d'Hippone, en affirmant l'universalité du bien, pense uniquement au bien ontique. Il y a lieu de comprendere ainsi son raisonnement: "Donc, tout ce qui est, est bon; et le mal, donc je cherchais l'origine, n'est pas une substance, parce que, s'il était une substance, il serait bon" ${ }^{17}$. Une autre énonciation est fondée sur le caractére universel du bien ontique. Ainsi: "Le bien donc peut être seul quelque part, le mal seul n'est nulle part. Les natures mêmes que leur mauvaise volonté a viciées dès l'origine, sont mauvaises en tant que viciées, mais bonnes en tant que natures» ${ }^{18}$. Nous y trouvons $\mathrm{l}^{\prime}$

13. J. Mausbach, Die Ethik des heiligen Augustinus (Freiburg 1909) t. I, p. 153; J. Hessen, Wertphilosophie (Paderborn 1937) p. 50-51. Hessen rejette l'adagium omne ens est bonum, en le considérant comme un produit de la philosophie scolastique.

14. En. I, 8,6; I, 8,13.

15. De Trinitate 15,5,8, PL 42,1062-1063; cf. S. Kowalczys, Dieu en tant que bien suprême selon l'acception de Saint Augustin, "Estudio Agustiniano" 6/1971/ p. 202.

16. De lib. arb. 2,18,48 PL 32,1266; 2,19,53 PL 32,1269.

17. Confes. 7,12,18. Bibliothèque Augustinienne. Oeuvres de St. Augustin, $t$. XIII. Trad. de E. Tréhorel et G. Bouissou (París 1962) Desclée de Brouwer, p. 621; PL 32,743.

18. De civ. Dei 12,3 Bibl. Augustinienne, t. XXXV. Trad. G. Combès (Paris 1959) p. 157; PL 41,351. 
affirmation explicite que toute nature est un bien ontique. Et même le mal moral apparaissant dans l'activité des êtres doués de raison, ne détruit pas le bien intrinsèque dans la nature des choses. Augustin se réfère à un exemple drastique du domaine de la théologie: dans l'acception de sa nature douée de raison, satan est un bien ontique. Ce qui, en lui, est mauvais, résulte de sa perversité morale qui abuse de la force et des qualités possédées par lui, pour des buts impropres ${ }^{10}$.

St. Augustin motive ainsi le caractère universel du bien ontique dans son oeuvre De vera religione:

"Quoniam quidquid est, quantulacumque specie sit necesse est; ita etsi minimum bonum, tamen bonum erit, et ex Deo erit. Nam quoniam summa species summum bonum est, minima species minimum bonum est) ${ }^{20}$.

Chaque être est un certain bien, s'il possède sa "species") déterminée. Ce terme apparaît avec plusieurs significations dans les écrits de notre penseur chrétien. Avant tout, il concerne l'aspect extérieur des choses: extérieur, formes harmonieuses, beauté. Toutefois, "species» désignait aussi la nature intérieure des choses; dans ce cas, le mot avait une signification apparentée à la notion de (eidos) ${ }^{21}$. De toute façon, il est certain que l'auteur de la citation considère le bien et l'être comme des idées ayant l'une et l'autre un domaine aussi vaste. L'universalité du bien résulte de l'universalité de l'être.

L'essentiel de l'argumentation augustinienne pour l'universalité du bien se ramène à l'affirmation que le bien est un phénomène positif, par comparaison auquel le mal n'est qu'un défaut secondaire. Cet aspect de l'axiologie de l'évêque d'Hippone est démontré, en abrégé, par la citation suivante, extraite des Confessiones: «Et il m'est clairement apparu que les choses qui se corrompent sont bonnes. Si elles étaient souverainement bonnes, ou si elles n'étaien nullement bonnes, ni dans un cas ni dans l'autre, elles ne pourraient se corrompre; car, souverainement bonnes, elles seraient incorruptibles; mais nullement bonnes, elles n'auraient pas en elles de quoi se corrompre. (...) Donc, si on la prive (une chose) de tout bien, elle ne sera plus rien. Donc, aussi longtemps qu'elle est, elle est bonne.

19. De civ. Dei $19,13,2$ PL 41,641 .

20. De ver. rel. 18,35 PL 34,137.

21. Cf. les observations de J. Pegon, BA t. VIII (Paris 1951), p. 486488. Cet auteur a traduit le terme latin de "species" par "type"; ibid., p. 71. 
Donc, tout ce qui est, est bon; et le mal, dont je cherchais l'origine, n'est pas une substance, parce que, s'il était une substance, il serait bon) ${ }^{22}$.

L'énonciation citée est dirigée contre le dualisme des manichéens qui admettaient l'existence de deux causes efficientes absolues de la réalité : le bien et le mal. Cela équivalait à l'absolutisation du mal. Augustin démontre l'absurdité de la métaphysique manichéenne, en insistant sur la nature privative du mal. Il se réfère à l'aperception intuitive de la réalité, dans laquelle nous apercevons en premier lieu l'être réel en tant que réalité positive ${ }^{23}$. Evidemment, nous voyons aussi la multiplicité du mal: matériel et moral, individuel et social, involontaire et prémédité. La mal est un fait indiscutable et général $^{2 *}$. Il est réel, mais d'une manière spéciale. Tout mal n'est qu'absence du bien obligatoire, son (comission) et sa "(corruption)" ${ }^{25}$. Le mal n'est pas (substance», donc il n'est pas positif ni autonome, il n'est que manque et déficit du bien ${ }^{26}$. Ainsi vue, la conception manichéenne du mal comme (anti-bien» positif, est un malentendu. Tout mal, en tant que défaut, doit avoir une base. Et celle-ci ne peut être que l'onticité réelle qui, en elle-même, est un bien. Augustin affirme paradoxalement que l'existence du bien est la condition de l'existence du mal. Le bien peut exister sans addition de mal, mais l'existence du mal en dehors du bien est impossible. "Toute nature corruptible est elle-même aussi un certain bien, car la corruption ne pourrait lui nuire si elle n'enlevait pas et ne diminuait pas ce qui est bien $)^{27}$. Le mal sort donc du bien, mais seulement d'un bien contingent, créé du néant.

Dans le contexte de la conception privative du mal, la thése des manichéens sur l'existence absolue et primordiale du mal, s'effondre. "Omnis autem natura in quantum natura est bonum est) ${ }^{28}$. Le bien

22. Confes. $7,12,18$. BA t. XIII, p. 619, 621; PL 32,743.

23. Dans le fragment cité, il a écrit: "Et il m'est clairement apparu...". Cf. "Quis enim dubitet totum illud quod dicitur malum, nihil enim esse quam corruptionem”. Contra ep. Manich. 35,39 PL 42,201.

24. Cf. De civ. Dei 19,2-7; Confes. 7,5,7; R. JolIVET, Le problème du mal chez Saint Augustin, éd. cit., p. 16 ss.

25. "Amissio boni mali nomen accipit". De civ. Dei 11,9 PL 41,325; "Nomenque hoc non sit nisi privatio boni". De civ. Dei 11,22 PL 41,335.

26. Cf. N. Blasquez, La idea de substancia en San Agustín (Madrid 1968) passim.

27. De $n$. boni 6 BA, t. I. Trad. B. Roland-Gosselin (Paris 1949), p. 447; PL 42,554.

28. De $n$. boni $1 \mathrm{PL} 42,551$. 
est donc l'aspect naturel de la réalité: il résulte autant de l'existence des choses que des attributs déterminés et de la perfection de la réalité. Le mal ne peut prétendre au rôle de co-principe causal du monde, car il ne présente qu'une spoliation de l'intégrité de l'être soit du bien. En parlant de la nature du mal, Augustin se réfère aux exemples concrets. La maladie n'existe qu'en tant que défaut de santé, l'infirmité n'est que manque d'intégrité du corps, la peur n'est qu' absence de courage et de calme ${ }^{29}$. Le mal n'est jamais un co-élément des qualités, mais une séquence de leur manque. Si le mal n'existe pas sans base de bien, l'existence du mal absolu ((pur») est intérieurement contradictoire. L'existence d'un néant intégral est impossible. Les manichéens considéraient la matière et les ténèbres comme un mal absolu. Augustin polémisait avec ces opinions en démontrant que les forces de la nature (l'eau, le feu) et les êtres individuels (les animaux, les humains) présentent un bien positif ${ }^{30}$. Le feu est un bienfait pour l'homme malgré que l'incendie entraîne des désastres pour lui. De même, les autres forces de la nature constituent, en elles-mêmes, un bien, ce n'est que lorsque l'ordre naturel ou l'integralité de l'être sont spoliés qu'arrive le mal.

Le bien est universel, mais, dans la sphère des êtres contingents, le mal est son corrélat. Le mal existe en deux variétés qui sont le mal ontique et le mal moral. Tout mal est un non-être relatif, une certaine disproportion axée sur la négation et le néant. Le mal ontique est un défaut involontaire et non intentionné, le mal éthique cependant est un guidage conscient et prémédité d'un être raisonnable vers le néant. “(...) Le péché ou l'iniquité ne soit pas un désir tourné vers des natures mauvaises mais un renoncement aux natures meilleures» ${ }^{31}$. Le mal éthique a donc une origine non ontique mais psychologique, sa source est dans l'abus du libre arbitre par une créature raisonnable. St. Augustin qualifie l'essence du mal moral de (mauvais emploi du bien) ${ }^{32}$. Ses analyses concernant les origines du mal autorisent à affirmer qu'il n'avait pas été porte-parole d'un optimisme naiif. Il admettait la réalité du mal, mais simultanément il considérait le bien comme primordial et fondamental.

29. Contra ep. Manich. 31,34 PL 42,197.

30. Contra ep. Manich. 1,31,34 PL 42,197. Cf. A. A. Moon, The De natura boni of Saint Augustine, éd. cit., p. 33-37.

31. De $n$. boni 34 BA, t. I, p. 477; PL 42,562.

32. "Malum est enim male uti bono". De $n$. boni 36 PL 42,562; "Deficitur enim non ad mala, sed male, id est non ad malas naturas, sed ideo male, quia contra ordinem naturarum". De civ. Dei 12,8 PL 41,355. 


\section{Les éléments du bien ontique}

Les écrits de St. Augustin ne contiennent pas de définition du bien, ni non plus de distinction formelle des éléments composant le bien ontique. Néanmoins, beaucoup de déclarations indiquent qu'il liait le bien ontique à la possession d'une existence réelle, et d'une nature spécifiquement définie, aisi qu'au désir du bien en tant que but. Le bien se compose donc de trois éléments: I.) existence, 2.) nature de la chose, 3.) actes de désir (d'amour).

Le bien ontique de toute chose commence par son existence. Le bien est un fait universel, et simultanément la valeur la plus essentie1le. De là, St. Augustin déduit sa conclusion: (In quanti sumus - boni sumus") ${ }^{33}$. Nombreuses sont ses énonciations sur l'identité de l' existence et du bien; ces opinions se trouvent autant dans ses écrits de jeunesse que dans ceux ultérieurs. En voici les plus caractéristiques: "Si (la chose) existe, elle est bonne; si elle ressent un manque d'existence, c'est qu'elle n'est pas issue de Dieu mais du néant) ${ }^{34}$. "Tous cependant, par le fait même de leur existence, sont à bon droit dignes d'être appréciés; car, par le fait même de leur existence, ils sont bons) ${ }^{35}$. "Mais si on prive une chose de tout bien, elle ne sera plus de tout. (...) Donc, aussi longtemps qu'elle est, elle est bonne) ${ }^{36}$. Ces déclarations autorisent plusieurs conclusions. En premier lieu, elles confirment le caractère universel du bien ontique. Quoiqu' il y ait des êtres, même raisonnables, qui ne sont pas éthiquement bons, tout est bon cependant dans l'aspect de l'existence. L'existence elle-même est un bien fondamental de chaque chose, car elle conditionne l'acceptation de toutes les autres valeurs. Les énonciations citées indiquent également que St. Augustin s'opposait à la séparation de la valeur et de l'être réel. $\mathrm{Vu}$ qu'il considérait l'existence comme le bien fondamental de toute chose, il y a lieu d'interpréter son axiologie en tant que métaphysique du bien et non comme une phénoménologie du bien. Pour cette raison, il serait difficile de considérer l' interprétation phénoménologique de la nature du bien comme une continuation fidèle de l'augustinisme. L'antique penseur chrétien

33. De civ. Dei 12,3 PL 41,351 .

34. Contra ep. Manich. 1,25,27 PL 42,191.

35. De lib. arb. 3,7,21 BA t. VI. Trad. F. J. Thonnard (Paris 1952) p. 363 ; PL 32,1281.

36. Confes. $7,12,18$ BA, t. XIII, p. 621 ; PL 32,743 . 
liait le bien organiquement à l'être; Max Scheler cependant admettait un dualisme de l'être et de la valeur ${ }^{37}$. Dans l'interprétation des phénoménologues, la valeur doit être autonome à l'égard de l'être tandis que, selon l'acception augustinienne, le bien résulte de l'être et de l'existence.

Tout en liant le bien à l'existence réelle, l'évêque d'Hippone tâchait d'expliquer la cause de leur union permanente. Pour lui, le bien réel, c'est-à-dire identique à l'être, est le seul bien authentique. La non-existence est négation et néant, et pourtant le néant ne peut contenir de valeur, c'est pourquoi on ne peut le choisir. A ce point de vue, l'homme qui voit son bonheur dans la non-existence, est dans l'erreur ${ }^{38}$. La non-existence, en tant que néant, ne peut constituer aucun bizn, puisque le bien est une valeur positive. Tout ce qui existe, désire conserver son existence ${ }^{39}$. Chez les êtres doués de raison, le désir d'exister est conscient, les animaux sont guidés par leur instinct, le monde des végétaux et des êtres inanimés possède un désir naturel de conservation de l'existence. L'homme souhaite poursuivre son existence au même degré qu'il veut son bonheur, puisqu'il serait bien difficile d'être heureux ayant cessé d'exister ${ }^{40}$.

La nature des choses présente le second élément du bien ontique. Le concept de la nature était compris par St. Augustin de diverses manières, et notamment comme: la nature divine, le cosmos, l'essence des choses ${ }^{41}$. La nature comprise dans ce dernier sens, embrasse l'ensemble des caractères génériques des choses ${ }^{42}$. A ce point de vue, les termes: natura, substantia, essentia sont des notions synonymes ${ }^{43}$.

37. "Alle Werte (auch die Werte 'gut' und 'bose'), sind materielle Qualitäten, die eine bestimmte Ordnung nach 'hoch' und 'nieder' zu einander haben, und dies unabhängig von den Seinsformen, in die sie eingehen". M. ScHELER, Der Formalismus in der Ethik und die materiale Wertethik (Halle 1927) p. 12.

38. De lib. arb. 3,8,22 PL 32,1281.

39. De civ. Dei 11,26 PL 41,340-341.

40. Tam porro nemo est qui esse se nolit, quam nemo est qui non beatus esse velit. Quomodo enim potest beatus esse si nihil est". De civ. Dei 11,26 PL 41,340-341.

41. Cf. De lib. arb. 3,19,54 PL 32,1297; Epist. 11,3 PL 33,76; F. J. TноNNARD, Notion de nature chez Augustin, "Revue des Etudes Augustiniennes" 11/1965/ p. 239-265.

42. Nam et ipsa natura nihil est alfud, quam id quod intelligitur in suo genere aliquid esse". De mor. Manich. 2,2,2 PL 32,1346.

43. "Omnis enim res, vel substantia, vel essentia, vel natura, vel st quo alio verbo melius enuntiatur". De ver. rel. 7,13 PL 34,129. Cf. N. BLASQUEZ, La idea de substancia en San Agustin, éd. cit. p. 38-40. 
Ces mots déterminent les éléments essentiels de l'être, les plus durables et les plus précieux. C'est ce qu'exprime la phrase de St. Augustin: "Toute nature, en tant que nature, est bonne" ". Le bien est échangeable avec l'être, mais il peut être exploité pour des fins moralement mauvaises. La tragédie de la creature raisonnable consiste en ce que, par abus de la liberté, (celle corrompt cette nature, faite bonnes " ${ }^{45}$. L'évêque d'Hippone, en motivant l'identité de la nature et du bien, insiste sur le fait que la dégradation de la nature de l'objet entraîne simultanément la disparition du bien. La mer houleuse est dangereuse ( (mauvaise»), mais la nature de l'eau est un bien ontique. L'élimination graduelle des propriétés de l'eau entraîne la perte de su nature substantielle et simultanément, la disparition du bien ${ }^{46}$.

La nature des choses se lie à la possession d'une certaine forme qui, elle aussi, conditionne le bien ontique. Sur ce thème, voilà ce qu'Augustin écrit dans son traité De vera religione: "Aussi, même à supposer que le monde ait été fait d'une matière, cette matière a été tout entière faite du néant. En effet, même ce qui n'a pas encore reçu sa forme est, d'une certaine manière, ébauché pour la recevoir. Cette aptitude à la forme est un bienfait de Dieu, puisque sa possession est un bien. La simple aptitude à la forme est donc un certain bien et par conséquent l'auteur de tous biens, qui a donné la forme, a donné aussi la possibilité d'être formé. [...] Mais nul être ne réalise intégralement sa nature, sans l'intégrité de tous ses caractères spécifiques. Or, toute intégrité vient de celui d'où vient aussi tout bien. Donc, tout bien venant de Dieu, toute intégrité vient de Dieu) ${ }^{47}$. L'auteur réfère ici la théorie chrétienne du créationnisme et, en marge, il confirme l'union organique du bien ontique et de la forme des choses. La forme qui actuellement façonne la matière est un bien supérieur; la matière seule capable de prendre forme est un bien inférieur. Dans l'acception augustinienne du bien, on perçoit l'écho de l'antique formule grecque: la forme est acte et perfection ${ }^{48}$. Il est cependant malaisé de préciser la conception de la forme chez l'évêque d'

44. Contra ep. Manich. 1,33,36 PL 42,199. Cf. Plotin, En. VI, 7,25.

45. [...] sed eam, quae bona condita est, vitiat". De gr. Chr. 19,20

PL 44,370 .

46. Contra ep. Manich. 1,33,36 PL 42,199.

47. De ver. rel. 18,36 BA, t. VIII. Trad. J. Pegon, (Paris 1951), p. 71,

73; PL 34,137. Cf. Enar. in ps. 102,8 PL 37,1322.

48. Cf. Platon, Phil. 22b; ArIStote, Metaph. XIV/N/, 1091 b 29. 
Hippone. E. Gilson suggère que le terme latin (forma) est une traduction du nom grec «idea». Il én résulte la nécessité d'interpréter la conception augustinienne de la forme dans le contexte de la théorie platonienne de participation. La forme des choses serait donc une participation aux idées éternelles de Dieu; grâce à cela, elle participe à la perfection de l'Absolu. Selon l'avis de Gilson, cidea, forma, species, ratio sont-ils finalement chez lui [Augustin] des termes synonymes” " Il semble que St. Augustin, dans sa conception de la forme, ait uni des éléments aristotéliciens et des éléments platoniens. Il est certain cependant qu'Augustin considérait la forme comme un facteur actif de l'être et qu'elle confère à l'être sa structure intérieure, sa petfection, son intégrité, ses caractères qualitatifs. Vu cette conception, de la forme, on arrive à comprendre la thèse de St. Augustin que la forme des choses est un facteur intégral du bien ontique.

La forme de l'être lui confère: le mode (modus), l'espèce (species) et l'ordre (ordo). L'action de la forme est interprété dans le traité De natura boni, comme la formation du bien ontique: "Là où le mode, la forme et l'ordre sont grands, les biens sont grands; où ils son petits, les biens sont petits; où ils sont nuls, il n'y a aucun bien. Et donc là où ils sont grands, les natures sont grandes; où ils sont petits, les natures sont petites; où ils sont nuls, il n'y a aucune nature. En conséquence, toute nature est bonne ${ }^{50}$. En parlant de modus, species et ordo, Augustin s'inspirait probablement des Ennéades de Plotin qui avait vu le bien de la chose en son unité, son espèce et son essence ${ }^{51}$. Toutefois, que signifient les termes mentionnés dans le De natura boni? Sans nul doute indiquent-ils l'action de la forme. L'un de ses effets est le modus, c'est-à-dire la limitation intérieure des perfections de la chose ${ }^{52}$. Un autre résultat de l'action de la forme est l'obtention par l'être de sa species. Ce concept a été emJloyé fréquemment par Augustin, avec des significations diverses: une fois, il signifiait l'extérieur de la chose, sa "figure) et ses caractéristiques; une autre fois, il s'agissait de la nature intérieure de l'

49. E. Gilson, Introduction à l'étude de Saint Augustin, (Paris 1949), p. 260. Cf. ibid., p. 256 274. Certaines formulations de St. Augustin confrment la suggestion de Gilson; cf. Confes. 13,2,2, PL 32,329; Div. quaest. oct. $\operatorname{tr}$. 46,2 PL 40,30 .

50. De $n$. boni $3 \mathrm{BA}$, t. I, p. 443,445; PL 42,553.

51. Plotin, En. I, 7,2; E. BREHIER, La Philosophie de Plotin (Paris 1928), p. 91.

52. Cf. De beat. vita 2,7 PL 32,963; Gén. ad litt. 4,3,7 PL 34,299. 
être, de son essence spécifique ${ }^{53}$. Le nom (species) compris en sa seconde signification, est synonyme du concept de forme ${ }^{54}$. Les deuz termes - species et forma- ont la même signification que le (eidos») grec, il s'agit notamment de l'essence de l'être soit de l'ensemble des caractéristiques de l'espèce. Enfin, le terme (ordo" indique la finalité de l'être, comprise en tant que l'ordre intérieur de ses éléments composants ${ }^{55}$. La forme de la chose lui conférant (mode), (espèce» et "ordre), fait le bien de chaque être. L'importance du bien ontique dépend avant tout de la qualité de la forme, c'est ainsi que les êtres animés sont plus parfaits que les inanimés, les êtres raisonnables plus parfaits que les irraisonnables, et les esprits purs plus parfaits que l' homme ${ }^{56}$. La plus bas degré de bien est attribué à la matière amorphe, n'ayant pas de forme. Si cependant les manichéens considéraient la matière comme l'incarnation du mal, St. Augustin par contre, la voyait comme un bien ontique puisquayant l'aptitude de prendre forme ${ }^{57}$.

Dans l'interprétation augustienne, l'existence réelle et la forme (la nature) des choses sont les éléments qui façonnent positivement le bien ontique. Selon l'opinion générale des hommes, chaque bien est lié au fait qu'il présente un objectif désirable pour quelqu'un. C'est ainsi qu'Aristote comprenait le bien, en le définissant comme id quod est appetibile ${ }^{58}$. Dans ses Ennéades, Plotin accepte cette définition du Stagyrite ${ }^{59}$. Chez St. Augustin, on ne trouve pas de répétition verbale de la définition aristotélicienne du bien, néanmoins, il lie souvent la notion de bien au fait de désir ou d'amour. Et voici une constatation caractéristique: "Avec cela, un bien qu'on n'aime pas, on ne saurait le posséder ou le connaître parfaitement: qui peut en effet apprécier un bien dont il ne jouit pas? Et il n'en jouit pas s'il ne l'ai-

53. De ver. rel. 11,21; 18,35; J. PEgot, Species, forma dans: BA, t. VIII, p. 486-488; R.H. NAsH, St. Augustine on Man's Knowledge of the Forms, "New Scholastism" 41/1967/ p. 223-234.

54. B. Roland-Gosselin, en traduisant De natura boni (BA, t. I, p. 443) interprète le terme latin "species" par le mot français "la forme", ce qui n'est pas une traduction adéquate, puisqu'il s'agit plutôt de l'essence spécifique de l'être.

55. Contra Secund. 10 PL 42,586. Cf. W. J. Roche, Measure, Number and Weight in Saint Augustine, "The New Scholasticism" 15/1941/. p. 350376; E. Gilson, op. cit., p. 186-187; A. A. Moon, op. cit., p. 126-128, 169.

56. De $n$. boni 5 PL 42,553 .

57. De $n$. boni 18 PL 42,556; A. T. RIPLINGER, Prime Matter in the Philosophy of St. Augustine, "Reality" /1962/, p. 51-70.

58. ARISTOTE, Eth. Nic. I, 1,1094 a 3.

59. Cfr. Plotin, En. I, 7,1; I, 8,2. 
me: aussi ne possède-t-il pas non plus ce qu'il doit aimer, celui qui ne l'aime pas, bien qu'on puisse aimer sans posséder' ' ${ }^{60}$. Le bien ne peut se passer d'amour et cela concerne avant tout le bien éthique. L'amour est l'attribut inséparable du bien et, en un certain sens, il présente la source et la base de celui-ci; la dynamique du bien est exprimée par la belle parole d'Augustin: "Charitas est radix bonorum ${ }^{61}$. Toutefois, Augustin avait la conviction que l'amour s'adresse au bien déjà existant ${ }^{62}$. Le bien comportant en soi une réelle perfection, devient objet de désir. Le phénomène du désir et de l'amour est aussi général que le bien ontique. Tous les êtres désirent quelque chose: la matière veut la forme, les végétaux souhaitent prolonger leur existence, les animaux désirent se nourrir, l'homme veut atteindre les valeurs supérieures ${ }^{63}$. St. Augustin attire l'attention sur ce que l'amour humaine doit s'acroître conformément au rang du bien désiré. Aussi doit-on infiniment aimer le bien infini ${ }^{64}$. L'amour du bien, quoique secondaire par rapport à la valeur objective de celui-ci, constitue son attribut indispensable. Le bien ontique est toujours objet de désir.

\section{La hiérarchie des biens}

En affirmant le caractère universel du bien ontique, St. Augustin percevait simultanément l'extrême abondance de formes et de degrés de ce bien. Il insistait aussi sur ce que la hiérarchie des biens n'est pas une invention de l'homme, mais que par contre, elle a un caractère objectif et naturel. Il définissait le mal moral comme infraction à l'ordre naturel des valeurs et la vertu comme respect de cet ordre ${ }^{65}$. Quelle est donc la hiérarchie des valeurs? Dans les oeuvres du penseur on trouve plusieurs classifications des biens. Elles sont plus ou moins développées, selon la problématique discutée. Dans le livre VIII du De Trinitate, il est question de Dieu en tant que vérité et

60. Div. quaest. oct. tr. BA, t. X. Trad .G. Bardy, (Paris 1952) p. 103; PL 40,24.

61. De gr. Chr. 1,20 PL 44,370.

62. "Nihil enim aliud est amare, quam propter se ipsam rem aliquam appetere". Div. quaest. oct. tr. 35,1 PL 40,24.

63. Trin. $8,3,4$ PL 42,949 .

64. Sermo 21,2-4 PL 38,138-141.

65. Cf. De n. boni 34 PL 42,562; De civ. Dei 12,8 PL 41,355; "Definitio brevis et vera virtutis, ordo est amoris". De civ. Dei 15,22 PL 41,467. 
bien suprêmes; c'est alors qu'Augustin a distingué une riche échelle de valeurs ${ }^{66}$ comprenant les types de biens suivants: les êtres inanimés (corps célestes, minéraux), les créatures vivantes (plantes, animaux), l'homme, créature douée de pensée, les esprits purs, enfin Dieu. La personne humaine, en tant qu'alliance de la matière et de l'esprit, joue un rôle crucial dans la hiérarchie des valeurs. Parmi les valeurs directement liées à la nature humaine, l'auteur distingue deux groupes: les biens vitaux et sensoriels qui servent le corps de l'homme, et les biens concernant l'âme humaine. Ceux-ci comprennent les biens esthétiques (la beauté, la poésie), ceux intellectuels (la science, la sagesse) et éthiques (l'amitié, la bonté). L'amour qui se situe plutôt au niveau surnaturel, est la couronne de toutes les valeurs.

L'homme est le point de répère de la classification des biens, présentée dans le De civitate $D e i^{\overline{6}}$. St. Augustin y a distingué trois types de biens, dont ceux extrinsèques, accidentellement liés à l'homme (bona extrinseca) sont estimés le plus bas, par exemple l'argent, les terres, la gloire, et les honneurs. Les biens servant directement le corps humain, telles la santé, la vigueur physique, l'agilité, les vicissitudes émotionnelles et sensuelles, appartiennent au second groupe. Ces valeurs ne présentent pas de mal, mais elles doivent être régies par les vertus de prudence et de tempérance ${ }^{68}$. Les valeurs vitales et sensorielles doivent être subordonnées aux valeurs spirituelles, ainsi que le corps - pars inferior de la nature humaine- est subordonné au bien de la personne. Il faut estimer avant tout les biens liés à l' élément spirituel de l'homme, donc les valeurs esthétiques, mentales et cognitives, éthiques et religieuses. Augustin fait remarquer que les valeurs spirituelles particulières ont un caractère partitif, ainsi les unes ne peuvent en remplacer d'autres. Et par exemple, la science n'observant pas les normes morales, conduit à la présomption ${ }^{69}$, aussi doit-elle être complétée par la sagesse qui confère à l'homme une lumière spéciale grâce à laquelle il s'élève des choses temporelles aux éternelles ${ }^{70}$. Dans la vie humaine, indispensables sont les vertus, habiletés morales positives et aptitudes à vivre une vie bonne, basée sur l'amour "t.

66. Trin. 8-3-4 PL 42,949-950.

67. De civ. Dei 8,8 PL 41,232-233; 19,3 PL 41,625; P. DELHAYE, Saint Augustin et les valeurs humaines, "Mélanges des sciences religieusés", $12 / 1955 /$, p. 121-138.

68. Sermo 51,13,21; PL 38,345; De bono matr. 19-22 PL 40,388-392.

69. Confes. 10,23 PL 32,793; De civ. Dei 11,27 PL 41,823.

70. Trin. 12,15,25 PL 42,1012; De lib. arb. 2,16,41 PL 32,1363.

71. "Recta ratio est ipsa virtus". De util. cred. 12,27 PL 42,85. 
L'échelle chrétienne des valeurs distingue en outre les valeurs s'unissant à la vie surnaturelle et qui sont: la foi, l'espérance, l'amour et le salut éternel ${ }^{\text {72 }}$.

Le traité $D e$ natura boni comporte une typologie abrégée des biens. On y lit: "Toute nature est esprit ou corps. L'esprit immuable, c'est Dieu. L'esprit muable, c'est une nature créée mais meilleure que le corps") ${ }^{73}$. C'est donc un classement des biens à trois termes, fondé sur la distinction entre le monde de la matière et celui de l'esprit, ainsi qu'entre le monde des créatures et Dieu. Nous trouvons cependant un autre caractère dans la typologie des valeurs mentionnée dans le De libero arbitrio, au début de l'argument sur l'existence de Dieu, déduit de l'éternité de la verité ${ }^{74}$. Augustin y signale trois niveaux d'existence: existence des minéraux (esse), la vie des plantes et des animaux (vivere), la pensée de l'homme (intelligere). On observe une différenciation pareille, mais plus ample, dans une autre opinion sur la vie de l'homme: "L'homme existe comme les arbres et les pierres, vit comme les plantes, sent comme les animaux, pense comme les anges) ${ }^{75}$. C'est là la constatation de l'existence de cinq niveaux de valeurs, et notamment: l'existence des êtres inanimés, la vie biologique des plantes, la vie sensuelle des animaux, la vie sensuelle $t$ spirituelle de l'homme, la vie purement spirituelle des anges. Le bien suprême dont ce texte ne parle pas, est Dieu.

Les classifications des biens par St. Augustin, ci-dessus mentionées, ont le plus souvent un caratère dichotomique. Ceci est dû à l' influence de la philosophie grecque classique: autant Platon qu'Aristote distinguaient un monde matériel et spirituel, sensoriel et mental, Dieu et la nature ${ }^{76}$. Le christianisme accepta ce dualisme en introduissant en plus le niveau de la vie surnaturelle. L'évêque d'Hippone, dans sa classification des biens, réunit les éléments grecs et les éléments chrétiens, en accentuant le rôle crucial de la personne humaine dans le monde des valeurs. Ses différenciations axiologiques étaient souvent dictées par des raisons pastorales, ainsi elles ne sont pas toujours entièrement correctes dans leur aspect théorique et mé-

72. De civ. Dei 19,4 PL 41,627 .

73. De $n$. boni I BA, t. I, p. 441; PL 42,551.

74. De Lib. arb. 2,3,7 PL 32,1243.

75. Sermo 43,3,4 PL 38,255.

76. Cfr. F.-J. von RINTELEN, Deus bonum omnis boni, dans: Aurelius Augustinus, éd. cit., p. 212-215. 
thodique ${ }^{77}$. En tout cas, pour résumer, on trouve chez l'antique penseur chrétien la différenciation des degrés de valeur suivants: l'existence des êtres matériels, la vie biologique des plantes, la vie sensuelle et instinctive des animaux, la vie mentale de l'homme, la vie purement spirituelle des anges, la vie Divine. Augustin discutait plus amplement les valeurs liées à l'homme, en distinguant dans ce domaine les valeurs cognitives, ethiques, esthétiques et religieuses.

La typologie augustinienne distingue les valeurs (supérieures») et (inférieures). Une question se pose: quel est le fondement de l' estimation des biens en particulier. Dans les oeuvres du penseur, on trouve plusieurs critères d'appréciation. L'un parmi ces critères est le critère anthropologique: les diverses valeurs sont définies en relation à l'homme, ainsi ce qui est bien suprême pour l'homme, ne l'est pas pour l'animal ${ }^{78}$. Souvent, le critère ontologique se présente, délimitant le monde de la matière et celui de l'esprit. Les valeurs liées à la sphère de la vie spirituelle (vérité, bien, amour) sont classées comme supérieures aux valeurs de la vie biologique ${ }^{79}$. La durabilité ontique et l'immuabilité des valeurs est un principe apparenté d'estimation, aussi faut-il apprécier les valeurs spirituelles plus hautement que celles émotionnelles et sensitives ${ }^{80}$. Le corps matériel est "périssable», l'âme cependant est immortelle. Toute créature est soumise à la loi de mutabilité, Dieu seul est absolûment immuable. Par conséquent, les biens spirituels et religieux, en tant que plus durables, son ontiquement supérieurs aux biens matériels et biologiques, muables et de courte durée. Chez Augustin, on trouve également le critère d'ampleur de l'étendue des valeurs; dans cet aspet, il jugeait la cognition intellectuelle supérieure à celle par les sens ${ }^{81}$. Cette dernière est partielle, cependant la cognition par l'intellect est plus universelle. Un autre critère d'estimation des biens résulte, chez Augustin, de l'affirmation d'un eudémonisme modéré. Supérieures sont donc les valeurs qui donnent à homme plus de bonheur durable ${ }^{82}$. Dans cet aspect, il y a lieu d'apprécier les valeurs spirituelles comme supérieu. res aux valeurs matérielles et sensorielles. Celles-ci réjouissent l'hom-

77. Cela concerne surtout les confrontations des valeurs de l'ordre de la nature et de l'ordre de la grâce.

78. De civ . Dei 19,3 PL 41,625.

79. Op. cit. 8,8 PL 41,231 .

80. De $n$. boni 5 PL 42,553 .

81. Ce critère est à la base de l'argument pour l'existence de Died déduit de l'éternité de la vérité.

82. De civ. Dei 8,8 PL 41,233. 
me, mais ce sont les premières qui forment sa personne. Le dernier critère axiologique avait été emprunté aux stoïques, et ensuite il fut inclus dans le monde chrétien des valeurs.

\section{Caractère de la théorie augustinienne $d u$ bien}

La théorie métaphysique du bien de St. Augustin, présentée en abrégé, permet cependant d'en tirer certaines conclusions. L'axiologie augustinienne est évidemment une expression d'optimisme, puisqu'elle constate le caractère universel du bien ontique. Le bien est une réalité primordiale, fondamentale et positive. Le mal, contrairement au pessimisme des Manichéens, ne posséde qu'une nature privative ${ }^{83}$. Le mal ontique est un résultat des limitations liées à la contingence de l'être créé. Le mal moral n'est pas d'origine ontique, mais psychologique; il résulte de l'abus du libre arbitre. Cela concerne aussi l'ange déchu, dont la nature spirituelle est un bien ontique. La recognition du fait du mal éthique préserve l'optimisme augustinien de la naiveté. Cet optimisme n'est qu'une apologie métaphysique de l' attitude de confiance adoptée par l'homme face au monde des valeurs qui l'environne.

Dans sa conception du bien, et en partie sous l'influence des Ennéades, St. Augustin refuse l'utilitarisme et l'hédonisme ${ }^{84}$. Cela se voit avant tout dans sa polémique contre le manichéisme, lorsqu'il établissait la différence entre le bien et l'utilité ${ }^{85}$. Ce qui est un bien objectif, n'est pas nécessairement utile à l'homme. Ainsi, les animaux sauvages possèdent beaucoup de caractères objectivement positifs (agilité, forces physiques) qui peuvent être dangereux pour un homme solitaire. La distinction entre le bien et le profit réapparaît dans les Confessiones. Nous y lisons: (Mais, dans les parties de la création, il y a certains éléments qui, par rapport à certains autres, ne conviennent pas, et pour cela sont estimés mauvais. Ces mêmes éléments conviennent à d'autres et sont bons, et en aux-mêmes ils sont bons) ${ }^{86}$. Cette énonciation confirme explicitement qu'Augustin était

83. Cf. A. VechI, L'antimanicheismo nelle Confessioni di Sant'Agostino, "Giornale di Metafisica", 20/1965/, p. 91-121.

84. Plotin, En. VI, 7,25.

85. Contra epist. Manich. 1,34,38 PL 42,200; 1,37,43 PL 42,203.

86. Confes. 7,13,19 BA, t. XIII, p. 621,623; PL 32,743. 
convaincu du caractère objetif du bien. Il voyait la source du bien dans la richesse ontique des choses et non pas dans l'estimation subjective ou l'avantage de l'homme.

L'évêque d'Hippone était le porte-parole du réalisme axiologique. Il considérait comme bien ce qui est lié organiquement à l'être, à son existence réelle et à sa nature définie dans son espèce. Les deux éléments composent les valeurs objetivement existantes, vers lesquelles se dirigent les actes de désir ou d'amour du sujet. Rintelen a observé judicieusement que la théorie de St. Augustin possède un cacactère métaphysique et non phénoménologique ${ }^{87}$. C'est ce qui la distingue des acceptions idéalistes du bien qui apparaissent dans le néokantisme et, en partie, dans la phénoménologie. Scheler et Hessen, dans leurs théories axiologiques, se sont éloignés du parfait réalisme de l'évêque d'Hippone qui voyait le bien avant tout dans l'objet, son existence et sa teneur. Les représentants allemands de la phénoménologie avaient une autre conception de la valeur: ils insistaient sur le rôle primordial du sujet et ils admettaient le dualisme de l'être et du bien ${ }^{88}$.

Un trait caractéristique de l'axiologie augustinienne est l'accentuation du caractère qualitatif du bien. C'est une interprétation qui diffère essentiellement des nombreuses acceptions contemporaines considérant l'aspect quantitatif du bien comme son élément fondamental: nombre, dimensions, fréquence. L'acception quantitative du bien est sensiblement l'effet du scientisme et du néo-positivisme, fascinés par l'évolution des sciences mathématiques et naturelles. St. Augustin interprète autrement le bien; il voit son essence dans l'as. pect qualificatif: possession de la forme, finalité de la structure de l' être, attributs spécifiques ${ }^{89}$. Le bien ontique est avant tout le "esse formatum). L'être est bon grâce à l'action de la forme qui, en formant la matière, lui confère son aspect extérieure ainsi que sont organisation intérieure. Dans cette acception du bien, on retrouve la continuation du platonisme et de l'aristotélisme qui considéraient la forme substantielle des choses comme leur richesse principale.

87. F.-J. VON RINTELEN, art. cit., p. 209.

88. M. SCHELER, Der Formalismus in der Ethik und die materiale Wertethik, (Halle 1927) p. 12-14,266; J. HESSEN, Wertphilosophie, (Paderborn 1937) p. 31,40,45-58,71-72. Il faut avouer que les deux auteurs ne proclamaient pas un subjectivisme de valeurs excessif, en admettant I' existence d'objets éléments đu bien.

89. De ver. rel. 18,36 PL 34,137; De n. boni 3 PL 42,553; F.-J. VoN RINTELEN, art. cit., p. 207-208. 
L'axiologie de St. Augustin avait un caractère existentiel. Toutefois, ce n'était pas un existentialisme du type vu par E. Gilson dans la méthaphysique de St. Thomas d'Aquin. L'Aquinate, en partant de principes systémo-métaphysiques, accentuait le rôle de l'existence en tant qu'acte de l'être. L'évêque d'Hippone évitait les schémas systématiques et les méditations spéculatives; il partait de l'analyse de l'existence humaine. Son existentialisme consistait dans l'alliance des considérations philosophiques concernant l'être et le bien, à la condition de la personnalité humaine. Il liait le bien à l'existence réelle de la chose, et sa nature spécifique, mais en même temps, il considérait le problème du bien et du mal en liaison avec l'existence humaine. L' existentialisme de la théorie augustinienne du bien se manifeste en son caractère anthropologique. On peut même parler d'un eudémonisme chrétien modéré, puisque le bien était analysé à travers l'inquietum cor de l'homme, chercheant le bonheur dans les valeurs transcendantes. L'existentialisme axiologique de l'évêque d'Hippone se manifestait également par l'emploi de la méthode du dialogue. C'était un dialogue de l'homme avec Dieu et de l'homme avec luimême. La concentration intérieure de l'homme -intellectuelle et éthique - est la condition inéluctable pour qu'il aperçoive le bien du cosmos qui l'environne ${ }^{n 1}$.

En donnant une caractérisque de la théorie du bien ontique chez St. Augustin, il faut attirer l'atention sur son théocentrisme. Celui-ci se manifestait par de nombreuses affirmations que les créatures participent au bien en Dieu comme Summum Bonum ${ }^{92}$. La perfection de l'être créé dépend du degré de son assimilation au Bien Suprême primordial ${ }^{93}$. Les minéraux ressemblent à Dieu par le fait de leur existence, les organismes par la possession de la vie, les humaines par la possession de l'intellect et de la volonté. Le théocentrisme axiologique de St. Augustin est lié à la théorie de causalité efficiente exemplaire. Par rapport à la théorie des valeurs, on en trouve une illus-

90. Cf. A. Solignac, L'existentialisme de Saint Augustin, "Nouvelle Revue Théologique" $70 / 1948 /$, p. 3-19; F. KORNER, Das Sein und der Mensch, éd. cit., p. 26-30. Les deux auteurs, en parlant de l'existentialisme de St. Augustin, pensaient avant tout à sa conception de l'être.

91. En ce qui concerne la vérité, F. M. Sciacca en parle amplement et d'une manière intéressante, en analysant l'argument augustinien pour l'existence de Dieu, partant de l'éternité de la vérité. Cf. L'Existence de Dieu, (Paris 1951).

92. De mor Manich. 2,4,6 PL 32,1347; S. CoNolly, The Platonisme of Augustine's "ascent" to God, "Irish Ecclesiastical Record" 78/1952/, p.4453.

93. "Nemo enim dubitat quod sit ipse primitus bonus. Multis enim 
tration dans l'énonciation suivante: “Ce n'est pourtant pas qu'il n'y ait en elle [en la créature] quelque ressemblance: qu'y a-t-il en effet qui, selon son degré d'être et selon sa mesure, n'ait une ressemblance avec Dieu, étant donné que toute oeuvre de Dieu est bonne (Eccli., XXXIX,2I), pour cette simple raison qu'il est lui-même le souverain Bien? Donc, dans la mesure où tout être est bon, il possède, encore que très lointaine, une certaine ressemblance avec le souverain Bien; naturelle, cette ressemblance est droite et dans l'ordre; viciée, elle est mauvaise et perverse') ${ }^{94}$.

Augustin suggère que le fondement du bien ontique se trouve dans le fait du reflet des perfections de Dieu dans la nature des créatures. Toute chose Lui ressemble à un certain degré, mais c'est une ressemblance imparfaite et partielle. La diversité ontique et axiologique des créatures est grande, cependant chacune est un reflet du bien absolu de Dieu. En expliquant le fait de participation au Bien Suprême, l'antique penseur chrétien emploie une terminologie apparentée à la théorie platonienne de la minesis ${ }^{95}$. Plotin lui aussi expliquait la corrélation du Bien primordial (Unité) et du monde visible à l'aide des notions : modèle-copie ${ }^{96}$. C'est dans ce contexte qu'il faut comprendre l'affirmation que les créatures ressemblent à Dieu. Une telle ressemblance se réalise également dans l'homme qui, grâce à son intellect, est l'image de Dieu la plus parfaite de toutes les créatures ${ }^{97}$. Entre Platon et Augustin, à côté de convergences terminologiques, il y a cependant d'importantes différences de système. Le premier réduisait le bien authentique au seul bien des idées dont les choses visibles n'étaient que (copies). Le penseur chrétien s'eloigna du platonisme extrême en accordant aux créatures un bien réel. Les créatures sont bonnes grâce à la possession de l'existence et d'une nature.

modis dici res possunt similes Deo: aliae secundum virtutem et sapientiam factae, [...] aliae in quantum solum vivunt, quis ille summe primitus vivit, aliae in quantum sunt, quia ille summe et primitus est". Div. quaest.oct.tr. 51,2 PL 40,32.

94. Trin. 11,5,8 BA, t.XVI. Trad. P. Agaësse, (Paris 1955), p. 185; PL 42,991 .

95. Platon, Timaios 29a; la République VI, 508c-509b; D. Ross, Plato's Theory of Ideas, (Oxford 1951) p. 221-224.

96. Plotin, En I,7,1; 3,5,1; 5,3,13.

97. "Inqua ipsa ratione, quae partim contemplativa est, partim activa, procul dubio contempatio praecellit. In hac enim et imago Dei est". Contra Faust. Man. 22,27 PL 42,418. Cf. M. Scmuaus, Die psychologische Trinitätslehre des heiligen Augustinus, (Münster 1927). 
Chez l'évêque d'Hippone le realisme axiologique s'allie au théocentrisme. Les créatures sont un bien ontique, mais simultanéament, elles dépendent de Dieu en tant que cause efficiente, modèle et finale. "Il y a un bien simple, Bien en soi-même, grâce à qui tout est un bien et de qui provient le bien ${ }^{98}$. Le bien des créatures lié à leur existence et à leur nature, vient finalement de Dieu en tant que leur cause suprême. La dépendance des créatures de Dieu fait que leur bien -autant dans l'aspect existentiel que dans l'aspect essentiel- est une participation Summi Boni ${ }^{99}$. Dans l'interprétation de St. Augustin, le théocentrisme axiologique n'enfreint ni la réalité ni l'authenticité du bien des créatures, il indique seulement leur contingence. Le bien ontique de l'homme et le bien du cosmos ne deviennent pleinement compréhensibles que lorsqu'on indique leur conditionnement transcendant. Le théocentrisme chrétien d'Augustin s'accorde harmonieusement, dans le domaine de la méthaphysique du bien, avec un anthropocentrisme modéré, en lui conférant des fondements durables et une dimension eschatologique.

\section{S. KowALCZYK}

Katolicki Uniwersytet Lubelski

98. Enar. in ps. 26,8 PL 36, 203. Cf. Doc chr. 1,32,35 PL 34,32; cf. S. Kowalczy , Dieu en tant que bien suprême selon l'acception de St. Augustin, "Estudio Agustiniano" 6/1971/, p. 199-213.

99. Confes. 13,2,2 PL 32,845; De ver rel. 18,36 PL 34,137. 